\title{
Die richtige Hämoglobinkonzentration beim kritisch kranken Patienten
}

\author{
Hans-Georg Bone
}

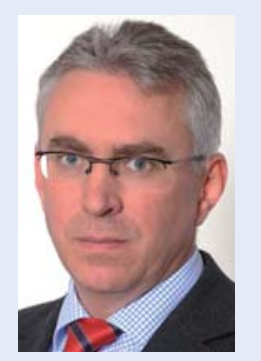

Hans-Georg Bone
Korrespondenzadresse Prof. Dr. med. Hans-Georg Bone Klinikum Vest GmbH Klinik für Anästhesiologie und IntensivmedizinKnappschaftskrankenhaus Dorstener Str. 151 45657 Recklinghausen Bone.Hans-Georg@KKRecklinghausen.de
Die Frage nach der richtigen Hämoglobinkonzentration beim kritisch kranken Patienten ist eigentlich beantwortet! Anders als in vielen anderen Ländern der Welt ist in Deutschland die Anwendung und die Gabe von Blutprodukten gesetzlich geregelt. Nach $\S 18$ des Transfusionsgesetzes stellt die Bundesärztekammer im Einvernehmen mit der zuständigen Bundesoberbehörde ... in Richtlinien den allgemein anerkannten Stand der medizinischen Wissenschaft und Technik ... für die Anwendung von Blutprodukten, einschließlich der Dokumentation der Indikation zur Anwendung von Blutprodukten ... fest. Es wird laut Gesetzt vermutet, dass der allgemein anerkannte Stand der medizinischen Wissenschaft ... eingehalten worden ist, wenn und soweit die Richtlinien der Bundesärztekammer ... beachtet worden sind. Die Bundesärztekammer hat aus diesem Grund Richtlinien erarbeitet und in diesen Richtlinien wiederum auf entsprechende Querschnittsleitlinien der Bundesärztekammer zur Therapie mit Blutkomponenten und Plasmaderivaten verwiesen. Die letzte Fassung dieser Querschnittsleitlinien stammt aus dem Jahr 2014. Auch wenn der Titel, Querschnittsleitlinien' etwas anderes vermuten lässt, handelt es sich bei dieser Publikation nicht um Leitlinien im klassischen Sinne, sondern, wie oben erläutert, eigentlich um Richtlinien mit einer sehr hohen Verbindlichkeit auf der Basis eines Gesetzes, sodass man als transfundierender Arzt schon gesetzlich gehalten ist, sich an diese Querschnittsleitlinien zu halten, damit der allgemein anerkannte Stand der medizinischen Wissenschaft beachtet wird. In diesen Leitlinien wird ausgeführt, dass die Gabe von Erythrozytenkonzentraten angezeigt ist, wenn Patienten ohne Transfusion durch eine anämische Hypoxie aller Voraussicht nach einen gesundheitlichen Schaden erleiden würden und eine andere, zumindest gleichwertige Therapie nicht möglich ist. Eine restriktive Indikationsstellung zur Erythrozytentransfusion ... geht bei den meisten Patientengruppen nicht mit einem erhöhten Mortalitätsrisiko einher [1]. Über Intensivpatienten heißt es in den Querschnittsleitlinien: Schwerkranke Patienten, die auf Intensivstationen überwacht und behandelt werden, können hin- sichtlich Morbidität und Mortalität von restriktiven Transfusionsstrategien, die Hämoglobinkonzentrationen zwischen 7 und 9g/dl als Zielwerte vorsehen, profitieren [1]. In den Querschnittsleitlinien wird empfohlen (diese ,Empfehlung hat, wie oben erläutert, eine hohe Verbindlichkeit), dass

1. wenn keine Risikofaktoren für eine eingeschränkte Kompensationsfähigkeit und keine Hinweise auf eine anämische Hypoxie vorliegen, bei einer Hämoglobinkonzentration von 6-8g/dl nicht transfundiert werden sollte.

2. beim Vorliegen einer eingeschränkten Kompensationsfähigkeit wie z. B. bei KHK, Herzinsuffizienz oder zerebrovaskulärer Insuffizienz bei einem Trigger von 6-8g/dl Hämoglobin transfundiert werden sollte.

3. beim Vorliegen von Hinweisen auf eine anämische Hypoxie bei Hb-Werten zwischen 6-10 g/dl transfundiert werden sollte. Hinweise auf eine solche anämische Hypoxie können u.a. sein: Tachykardie, Hypotension, Dyspnoe, EKG-Veränderungen, neu aufgetretene Veränderungen in der Echokardiografie, Abfall der zentralvenösen Sauerstoffsättigung auf unter $60 \%$ oder ein signifikanter Laktatanstieg.

4. bei Hb-Werten über $10 \mathrm{~g} / \mathrm{dl}$ nicht transfundiert werden sollte.

Damit wäre eigentlich alles klar: Laut Querschnittsleitlinien sollte auch beim kritisch kranken Patienten eine Transfusion zurückhaltend erfolgen und der Transfusionstrigger in Abhängigkeit von Vorerkrankungen und Zeichen akuter anämischer Hypoxie zwischen 6-10 g/dl liegen. Details zu Transfusionsindikationen und der Erythrozytenkonzentratgabe bei verschiedenen intensivmedizinischen Krankheitsbildern werden in dieser Ausgabe der Intensivmedizin up2date in der Übersichtsarbeit von Neumann, Campos und Unterberg diskutiert. Wie komplex manchmal dennoch die Indikationen zur Transfusion sind, bzw. wie schnell sich die wissenschaftliche Evidenz für solche Indikationen ändert, soll kurz an einem Beispiel erläutert werden: 
Im Jahr 2001 publizierten Rivers et al. eine monozentrische Studie zur frühen hämodynamischen Therapie bei septischen Patienten (Early goal directed therapy) [2]. In dieser Studie wurde gezeigt, dass eine frühe, anhand der zentralvenösen Sauerstoffsättigung gesteuerte Therapie, bei der in einem Bündel von Maßnahmen unter anderem eine Hämatokritkonzentration von $\geq 30 \%$ angestrebt wurde, mit einer reduzierten Mortalität einhergeht. Bei erniedrigten Werten der zentralvenösen Sättigung und Unterschreiten der angestrebten Hämatokritkonzentrationen in der Behandlungsgruppe wurden Erythrozytenkonzentrate transfundiert. Eine Begründung für das Auswählen speziell dieses Transfusionstriggers gaben die Autoren in der Publikation nicht. Rasch wurde dennoch für die nächsten 10 Jahre in alle wichtigen internationalen und nationalen Sepsisleitlinien dieser Transfusionstrigger von Hkt $\geq 30 \%$ in die Empfehlungen für die frühe Behandlung der schweren Sepsis und des septischen Schocks aufgenommen [3-6]. In den vergangenen letzten 18 Monaten haben dann aber 3 große multizentrische Studien keine Vorteile dieser ,Early goal directed therapy‘ zeigen können [7-9]. Sehr wahrscheinlich wird mit diesen Ergebnissen auch die Empfehlung für das sehr hohe Hämatokritziel bei der frühen Behandlung der Sepsis fallen. Nicht nur im Spezialfall der initialen Behandlung der schweren Sepsis, sondern auch für viele andere intensivmedizinische Szenarien gab es in den letzten Jahren erneut gute Evidenz, an der restriktiven Transfusionsstrategie festzuhalten. So wurden z.B. im großen skandinavischen TRISS Trial fast 1000 Patienten mit septischem Schock entweder einer restriktiven Transfusionsgruppe mit einem Transfusionstrigger von $7 \mathrm{~g} / \mathrm{dl}$ oder einer liberalen Transfusionsgruppe mit einem Transfusionstrigger von $9 \mathrm{~g} / \mathrm{dl}$ zugeordnet [10]. Zwischen den beiden Gruppen fanden sich keinerlei Unterschiede in Bezug auf die 90-TageMortalität und die Häufigkeit ischämischer Komplikationen. Die Patienten der liberalen Transfusionsgruppe erhielten aber ca. die vierfache Menge an Transfusionen. Anders als die deutlichen Ergebnisse bei kritisch kranken Patienten konnten die Vorteile einer restriktiven Transfusionsstrategie im perioperativen Setting nicht immer bestätigt werden, so ergab z. B. eine aktuelle, lebhaft diskutierte, multizentrische Studie bei kardiochirurgischen Patienten, dass eine restriktive postoperative Transfusionsstrategie im Vergleich zu einer liberaleren Strategie sogar mit einer erhöhten Mortalität einhergehen kann [11]. Selbst unterschiedliche Metaanalysen der bislang vorliegenden, weitestgehend gleichen Studien zum Thema ,Transfusionstrigger ergaben divergente Ergebnisse, mal war eine perioperative restriktive Transfusionsstrategie positiv und mal nicht $[12,13]$. Für die kritisch kranken intensivpflichtigen Patienten sind aber die Ergebnisse der Metaanalysen eindeutig: Diese Patienten profitieren von einer restriktiven Transfusionsstrategie. Die Querschnittsleitlinien der Bundesärztekammer haben das in ihren Empfehlungen schon lange berücksichtigt.

\section{Literatur}

1 Bundesärztekammer. Querschnitts-Leitlinien (BÄK) zur Therapie mit Blutkomponenten und Plasmaderivaten. 2014: 4. überarbeitete und aktualisierte Auflage

2 Rivers $E$ et al. Early goal-directed therapy in the treatment of severe sepsis and septic shock. N Engl J Med 2001; 345: $1368-1377$

3 Dellinger RP et al. Surviving Sepsis Campaign guidelines for management of severe sepsis and septic shock. Crit Care Med 2004; 32: $858-873$

4 Dellinger RP et al. Surviving Sepsis Campaign: international guidelines for management of severe sepsis and septic shock: 2008. Crit Care Med 2008; 36: 296 - 327

5 Dellinger RP et al. Surviving sepsis campaign: international guidelines for management of severe sepsis and septic shock: 2012. Crit Care Med 2013; 41: 580-637

6 Reinhart K et al. Prävention, Diagnose, Therapie und Nachsorge der Sepsis. Erste Revision der S2k-Leitlinien der Deutschen Sepsis-Gesellschaft e.V. (DSG) und der Deutschen Interdisziplinären Vereinigung für Intensiv- und Notfallmedizin (DIVI). Anaesthesist 2010; 59: 347 - 370

7 The ProCESS Investigators. A Randomized Trial of ProtocolBased Care for Early Septic Shock. N Engl J Med 2014; 370: $1683-1693$

8 The ARISE Investigators and the ANZICS Clinical Trials Group. Goal-Directed Resuscitation for Patients with Early Septic Shock. N Engl J Med 2014; 371: 1496 - 1506

9 ProMISe Trial Investigators. Trial of Early, Goal-Directed Resuscitation for Septic Shock. N Engl J Med 2015; 372: $1301-1311$

10 Holst LB et al. Lower versus higher hemoglobin threshold for transfusion in septic shock. N Engl J Med 2014; 371: 1381 1391

11 Murphy G] et al. Liberal or restrictive transfusion after cardiac surgery. N Engl J Med 2015; 372: 997 - 1008

12 Salpeter SR, Buckley JS, Chatterjee S. Impact of more restrictive blood transfusion strategies on clinical outcomes: a meta-analysis and systematic review. Am J Med 2014; 127 : $124-131$

13 Fominskiy E et al. Liberal transfusion strategy improves survival in perioperative but not in critically ill patients. A metaanalysis of randomised trials. Br J Anaesth 2015; 115: 511 519 